\title{
Lactosylated liposomes for targeted delivery of doxorubicin to hepatocellular carcinoma
}

This article was published in the following Dove Press journal:

International Journal of Nanomedicine

15 October 2012

Number of times this article has been viewed

\author{
Xiaoju Zhou ${ }^{1,2, *}$ \\ Mengzi Zhang,** \\ Bryant Yung ${ }^{2}$ \\ Hong $\mathrm{Li}^{2}$ \\ Chenguang Zhou ${ }^{2}$ \\ $L$ James $L^{2 e e^{3,4}}$ \\ Robert J Lee ${ }^{2,4}$
}

'State Key Laboratory of Virology, Ministry of Education Key Laboratory of Combinatorial Biosynthesis and Drug Discovery, Wuhan University School of Pharmaceutical Sciences, Wuhan, People's Republic of China; ${ }^{2}$ Division of Pharmaceutics, ${ }^{3}$ Department of Chemical and Biomolecular Engineering, ${ }^{4}$ NSF Nanoscale Science and Engineering Center for Affordable Nanoengineering of Polymeric Biomedical Devices, The Ohio State University, Columbus, $\mathrm{OH}$, USA

*These authors contributed equally to this work

Correspondence: Robert J Lee The Ohio State University, College of Pharmacy, Parks Hall 542, $500 \mathrm{~W}$ I2th Avenue, Columbus,

$\mathrm{OH} 43210$, USA

Tel +l 6142924172

$\mathrm{Fax}+\mathrm{I} 6142927766$

Email lee.1339@osu.edu
Background: N-lactosyl-dioleoylphosphatidylethanolamine (Lac-DOPE) was synthesized and evaluated as a liver-specific targeting ligand via asialoglycoprotein receptors for liposomal delivery of doxorubicin.

Methods: Lactosylated liposomes encapsulating calcein (Lac-L-calcein) or doxorubicin (Lac-L-DOX) composed of egg phosphatidylcholine, cholesterol, monomethoxy polyethylene glycol 2000-distearoyl phosphatidylethanolamine, and Lac-DOPE at 50:35:5:10 ( $\mathrm{mol} / \mathrm{mol})$ were prepared by polycarbonate membrane extrusion and evaluated in human hepatocellular carcinoma HepG2 cells. Cellular uptake of Lac-L-calcein was monitored by confocal microscopy and by flow cytometry. The cytotoxicity of Lac-L-DOX was evaluated by MTT assay. The pharmacokinetic properties of Lac-L-DOX were studied in normal mice, and its biodistribution and antitumor activity were studied in nude mice with HepG2 xenografts.

Results: The size of Lac-L-DOX was less than $100 \mathrm{~nm}$ and the liposomes demonstrated excellent colloidal stability. In vitro uptake of Lac-L-calcein by HepG2 cells was four times greater than that of non-targeted L-calcein. In the presence of $20 \mathrm{mM}$ lactose, the uptake of Lac-L-calcein was inhibited, suggesting that asialoglycoprotein receptors mediated the observed cellular uptake. Lac-L-DOX exhibited enhanced in vivo cytotoxicity compared with the nontargeted liposomal doxorubicin (L-DOX), and its pharmacokinetic parameters indicate that Lac-L-DOX has a long blood circulation time ( $\mathrm{t}_{1 / 2} 8.73$ hours). Tissue distribution and therapeutic efficacy studies in nude mice bearing HepG2 xenografts show that Lac-L-DOX had significantly stronger tumor inhibitory activity compared with L-DOX and free doxorubicin, along with a higher accumulation of drug within the tumor site and greater cellular uptake by tumor cells.

Conclusion: These data suggest that lactosylated liposomes are promising drug delivery vehicles for hepatocellular carcinoma.

Keywords: liposomes, hepatocellular carcinoma, asialoglycoprotein receptor, drug targeting

\section{Introduction}

Hepatocellular carcinoma is one of the most common types of cancer and is associated with a high rate of mortality. ${ }^{1}$ Doxorubicin (DOX) is an important chemotherapeutic agent for hepatocellular carcinoma. However, it is associated with dose-limiting cardiotoxicity. Liposomal delivery of DOX has resulted in reduced cardiotoxicity. ${ }^{2,3}$ Doxil, a PEGylated long circulating version of liposomal DOX (L-DOX), is approved for platinum-resistant ovarian carcinoma. However, it has shown limited efficacy against hepatocellular carcinoma. Liposomes are cleared by the reticuloendothelial system (RES) after intravenous administration and are taken up by nonparenchymal Kupffer cells in the liver. ${ }^{4,5}$ Because hepatocellular carcinoma originates from liver parenchymal 
cells, it is desirable to deliver drugs selectively to hepatocytes. To this end, asialoglycoprotein receptors (ASGPR) are frequently used as a target due to the high expression on the surface of hepatocytes and in hepatocellular carcinoma cell lines. Studies have shown that modification with D-galactose or $\mathrm{N}$-acetylgalactosamine residues effectively target drugs to hepatocytes via a pathway dependent on ASGPR. ${ }^{6-9}$ Several different lactosylated lipids have been synthesized for incorporation into liposomes and their targeting capacity to hepatic tumor cells has been reported. ${ }^{10-14} \mathrm{Xu}$ et $\mathrm{al}^{15}$ synthesized a lactobionic acid conjugate of dioleoylphosphatidylethanolamine (Lac-DOPE) for targeting of solid lipid docetaxel-loaded nanoparticles. The researchers found that the targeted nanocarrier enhanced the antitumor activity of docetaxel in hepatocellular carcinoma. In this study, Lac-DOPE was synthesized and incorporated into L-DOX (Lac-L-DOX). The cytotoxicity, uptake by HepG2 cells, and pharmacokinetic properties of Lac-L-DOX were evaluated. In addition, the distribution and antitumor efficacy of Lac-L-DOX was studied in nude mice with HepG2 xenografts.

\section{Materials and methods}

\section{Materials}

Egg phosphatidylcholine and DOPE were purchased from Avanti Polar Lipids (Alabaster, AL). Monomethoxy polyethylene glycol 2000-distearoyl phosphatidylethanolamine (mPEG-DSPE) was purchased from Genzyme Pharmaceuticals (Cambridge, MA). Cholesterol, DOX, lactobionic acid, lactose, Sepharose CL-4B chromatography resin, calcein, 3-(4,5-dimethyl-2-thiazolyl)-2,5-diphenyltetrazolium bromide (MTT), chloroform, and dimethyl sulfoxide were purchased from Sigma-Aldrich Chemical Company (St Louis, MO). Alexa Fluor 488® phalloidin was purchased from Life Technologies (Carlsbad, CA). 1-Ethyl-3-[3-dimethylaminopropyl] carbodiimide hydrochloride (EDC) and N-hydroxysuccinimide (NHS) were obtained from Thermo Scientific (Rockford, IL). Phosphate-buffered saline (PBS) was purchased from Fisher Scientific. All other reagents were of analytical grade and used without further purification.

\section{Synthesis of Lac-DOPE}

Briefly, a solution of $240 \mathrm{mg}$ lactobionic acid in $5.0 \mathrm{~mL}$ of PBS was added dropwise to a stirring solution of $100 \mathrm{mg}$ DOPE in $5.0 \mathrm{~mL}$ ethanol. A small amount of triethylamine was added into the mixing solution until the solution became clear. Next, $257.5 \mathrm{mg}$ EDC and $77.5 \mathrm{mg}$ NHS were added into the solution, and the reaction was allowed to proceed for 150 minutes at room temperature. The reaction scheme is shown in Figure 1. The product was purified by silica gel column chromatography using chloroform/methanol/water (3/1/0.15, v/v) for product elution. ${ }^{1} \mathrm{H}$ NMR analysis of Lac-DOPE (300 MHz, $\mathrm{CDCl}_{3}$ ) showed principal peaks of DOPE moiety to be $\delta 0.86(\mathrm{t}, 6 \mathrm{H})$, $1.25(\mathrm{~m}, 44 \mathrm{H}), 1.6(\mathrm{~m}, 6 \mathrm{H}), 1.99(\mathrm{~m}, 8 \mathrm{H}), 2.2(\mathrm{q}, 2 \mathrm{H}), 5.20$ $(\mathrm{s}, 1 \mathrm{H})$, and $5.36(\mathrm{~m}, 4 \mathrm{H})$, and that of the LA moiety to be $3.4-4.6$, which is consistent with a previous report. ${ }^{13}$

\section{Liposome preparation}

The liposomes were prepared by an ammonium sulfate-based $\mathrm{pH}$ gradient-driven remote-loading method as described previously. ${ }^{16,17}$ The lipid components of L-DOX were egg phosphatidylcholine to cholesterol to $\mathrm{MPEG}-\mathrm{DSPE}$ at a molar ratio of 60:35:5. The lipid components of the Lac-L-DOX

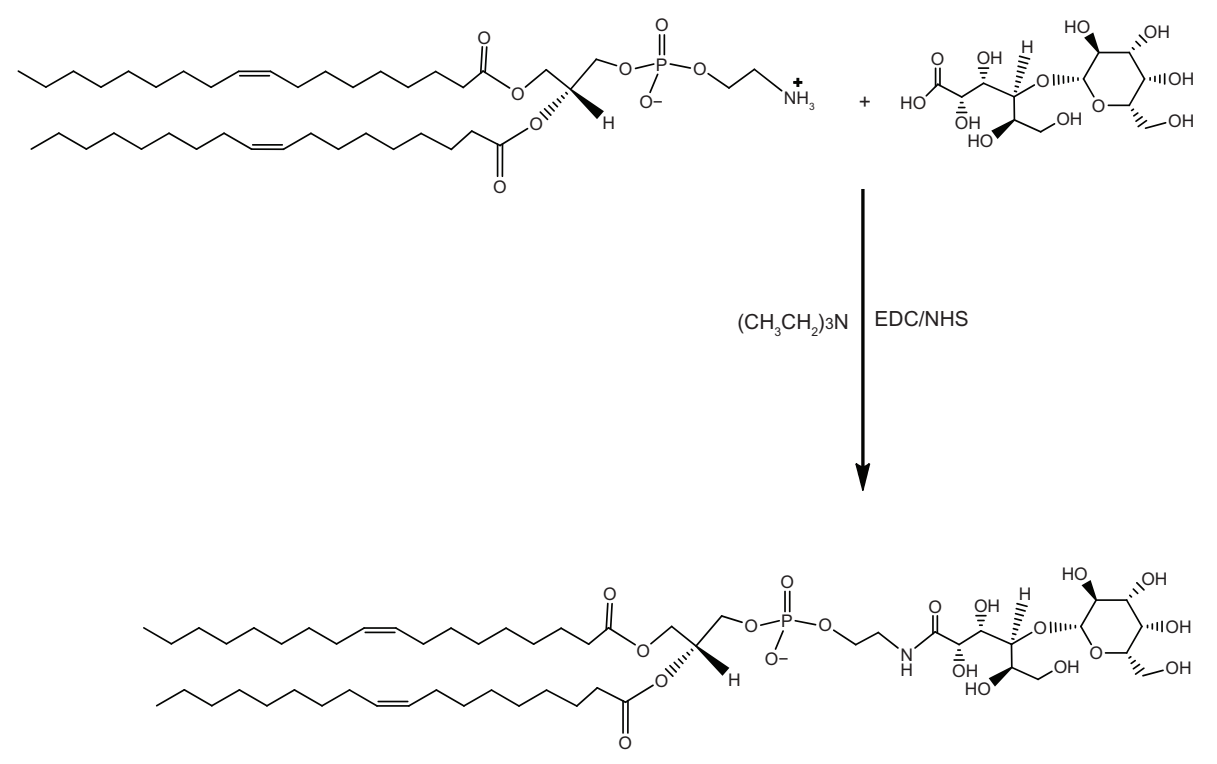

Figure I Synthesis of Lac-DOPE. 
were egg phosphatidylcholine, cholesterol, mPEG-DSPE, and Lac-DOPE at a molar ratio of 50:35:5:10. Briefly, the lipids were dissolved in chloroform and dried to a thin lipid film under rotary evaporation at $60 \mathrm{rpm}$ and $40^{\circ} \mathrm{C}$, followed by further drying under vacuum. The thin lipid film was hydrated with $250 \mathrm{mM}\left(\mathrm{NH}_{4}\right)_{2} \mathrm{SO}_{4}$ solution at room temperature and subjected to three cycles of freezing and thawing. The suspension of multilamellar vesicles was extruded three times through a $100 \mathrm{~nm}$ pore size polycarbonate membrane on a nitrogen-driven Lipex lipid extruder (Northern Lipids Inc, Vancouver, $\mathrm{BC})$. The external $\left(\mathrm{NH}_{4}\right)_{2} \mathrm{SO}_{4}$ of the liposomes was removed by tangential flow diafiltration against PBS (pH 7.4) using a Millipore Pellicon XL cartridge with a molecular weight cutoff of $30 \mathrm{kDa}$. DOX solution was then added to the empty liposomes and incubated at room temperature for 30 minutes. The unencapsulated DOX was removed by passing the liposome solution through a Sepharose CL-4B column. Calcein-loaded liposomes (L-calcein and Lac-L-calcein) were prepared by a polycarbonate membrane extrusion method, as described previously by Lee et al. ${ }^{18}$ The same lipid components as listed above were used.

DOX and calcein concentrations were determined by measuring absorbance at $480 \mathrm{~nm}$ and $495 \mathrm{~nm}$, respectively, on a Shimadzu ultraviolet-visible spectrophotometer (Tokyo, Japan). Particle size was determined by dynamic laser light scattering (submicron particle sizer, Nicomp ${ }^{\text {TM }}$ 370, Santa Barbara, CA). The zeta potential was measured using a ZetaPALS (zeta potential analyzer, Brookhaven Instruments Corporation, Holtsville, NY).

\section{In vitro experiment}

\section{Cell culture}

HepG2 cells were cultured in RPMI 1640 medium (Gibco, Grand Island, NY) supplemented with 10\% fetal bovine serum (Invitrogen), $100 \mathrm{U} / \mathrm{mL}$ penicillin, and $100 \mathrm{mg} / \mathrm{mL}$ streptomycin. Cells were maintained in a humidified atmosphere containing $5 \% \mathrm{CO}_{2}$ at $37^{\circ} \mathrm{C}$.

\section{In vitro cellular uptake of liposomal calcein}

HepG2 cells were seeded in a six-well plate at a density of $2.0 \times 10^{5}$ cells per well and cultured in RPMI 1640 medium for 24 hours. L-calcein, Lac-L-calcein, and Lac-L-calcein with $20 \mathrm{mM}$ lactose as an ASGPR blocking agent were added in equivalent calcein amounts per well and incubated for one hour. The cells were washed three times with cold PBS. Fluorescence was detected using a flow cytometer (Beckman Coulter Inc, Fullerton, CA). Cell-associated calcein was excited with the $488 \mathrm{~nm}$ line of argon, and emission fluorescence was measured between $564 \mathrm{~nm}$ and
$606 \mathrm{~nm}$. For each sample, data for individual fluorescence of 10,000 cells were collected.

\section{Subcellular localization of liposomal calcein}

HepG2 cells were incubated with L-calcein, Lac-L-calcein, and Lac-L-calcein with $20 \mathrm{mM}$ lactose in RPMI 1640 medium for one hour on round glass coverslips measuring $14 \mathrm{~mm}^{2}$ in culture dishes. The cells were imaged with a Flowview 1000 laser confocal scanning microscope (Olympus, Tokyo, Japan) equipped with a $60 \times$ oil immersion lens. The excitation/emission wavelengths used were $488 \mathrm{~nm} / 577 \mathrm{~nm}$ for the liposomes.

\section{In vitro cytotoxicity assay}

The in vitro cytotoxicity of DOX-loaded liposomes was evaluated by MTT assay. ${ }^{19-21}$ Briefly, HepG2 cells $\left(5.0 \times 10^{3}\right.$ cells/100 $\mu \mathrm{L}$ per well) were seeded into 96 -well plates in RPMI medium supplemented with $10 \% \mathrm{v} / \mathrm{v}$ fetal bovine serum and $1 \%$ penicillin-streptomycin. Cells were incubated with $100 \mu \mathrm{L}$ of 1:4 serial diluted free DOX, L-DOX, Lac-L-DOX, or Lac-L-DOX with $20 \mathrm{mM}$ lactose as an ASGPR-blocking agent $(0,0.0625,0.25,1,4,16,64,256$, and $1024 \mu \mathrm{M}$ DOX, respectively) for 2 hours at $37^{\circ} \mathrm{C}$. After incubation, the cells were washed three times with cold PBS and cultured for an additional 72 hours in $200 \mu \mathrm{L}$ of fresh medium. Next, $20 \mu \mathrm{L}$ of MTT $5 \mathrm{mg} / \mathrm{mL}$ was added to each well, followed by incubation for 4 hours at $37^{\circ} \mathrm{C}$. The formazan crystals were dissolved in $200 \mu \mathrm{L}$ of dimethyl sulfoxide. The absorbance was determined at $570 \mathrm{~nm}$ on a Dynatech MR-600 microplate reader (Bio-Rad, Hercules, CA).

\section{Animal experiments Animals}

Female imprinting control region mice (aged 5-6 weeks, weighing 18-20 g) and athymic nude-Foxn1nu mice (5-6 weeks, 18-20 g) were purchased from Harlan Laboratories (Indianapolis, IN). All experimental procedures were done according to protocols approved by the Ohio State University Institutional Laboratory Animal Care and Use Committee.

\section{Pharmacokinetics of DOX in mice}

Free DOX, Lac-L-DOX, or L-DOX was administered to imprinting control region mice through tail vein injection at a dose of $5 \mathrm{mg} / \mathrm{kg}$. This study used groups of three mice per liposome formulation per time point. At the time points of $0.25,0.5,1,2,4,8,16$, and 24 hours, blood samples were collected in heparinized tubes via heart puncture and centrifuged at $4000 \mathrm{rpm}$ for 10 minutes. The concentrations of DOX in plasma were assayed by a spectrofluorometric method previously described in the literature. ${ }^{22,23}$ Briefly, 
$10 \mu \mathrm{L}$ of plasma was diluted with $990 \mu \mathrm{L}$ of acidic isopropanol (81 $\mathrm{mM} \mathrm{HCl}$ in isopropanol) and incubated at $4^{\circ} \mathrm{C}$ overnight protected from light. The sample was then centrifuged for 10 minutes at $12,000 \times \mathrm{g}$ and the supernatant was assayed in a spectrofluorometer (Hitachi 650-60, Tokyo, Japan) at an excitation wavelength of $485 \mathrm{~nm}$ and an emission wavelength of $590 \mathrm{~nm}$.

\section{Tissue distribution of DOX in tumor-bearing mice}

HepG2 cells $\left(5.0 \times 10^{6}\right.$ cells $\left./ 100 \mu \mathrm{L}\right)$ were injected subcutaneously into the left flank of female athymic nude mice. The experiment was performed on day 7 after tumor cells were implanted. Tumor-bearing mice were randomly divided into one of three groups (five per group) and injected with DOX, L-DOX, or Lac-L-DOX at a single dose of $5 \mathrm{mg} / \mathrm{kg}$ via the tail vein. At 4 hours after drug administration, the mice were sacrificed, and the tumor, liver, kidneys, spleen, lungs, and heart were collected. The tissue samples were washed with PBS and excess fluid was removed. The DOX content in the tissues was determined as described in the literature. ${ }^{24}$ Briefly, $0.1-0.3 \mathrm{~g}$ of the tissue samples were suspended in $1.5 \mathrm{~mL}$ nuclear lysis buffer (10 mM HEPES, $1 \mathrm{mM} \mathrm{MgSO}$, $1 \mathrm{mM} \mathrm{CaCl}_{2}$, $\mathrm{pH}$ 7.4) and homogenized using a Tissue-Tearor homogenizer (BioSpec Products Inc, model 985370, Bartlesville, OK). The homogenate $(100 \mu \mathrm{L})$ was added to $50 \mu \mathrm{L}$ of $10 \%(\mathrm{v} / \mathrm{v})$ Triton $\mathrm{X}-100,100 \mu \mathrm{L}$ of water, and $750 \mu \mathrm{L}$ acidified isopropanol. The mixture was then stored overnight at $-20^{\circ} \mathrm{C}$, followed by centrifugation for 10 minutes at 14,000 rpm after thawing. The supernatant was subsequently analyzed by fluorescence microscopy (Olympus BX-51, Tokyo, Japan).

\section{Tissue confocal imaging studies}

The livers and tumors above were processed as described previously. ${ }^{25}$ Briefly, tissues were fixed using 3\% paraformaldehyde solution in PBS at $4^{\circ} \mathrm{C}$ for 30 minutes, washed with PBS, and immersed in 30\% sucrose solution. The processed tissue was then embedded in paraffin and frozen in liquid nitrogen. The tissue blocks were cut on a Reichert-Jung cryostat at a section thickness of $7 \mu \mathrm{M}$. For actin staining, the slides were incubated with Alexa Fluor 488 phalloidin for 20 minutes. The fluorescence distribution in each tissue was observed by laser confocal scanning microscopy (Nikon Bioscience Confocal Systems, Melville, NY).

\section{Antitumor activity in vivo}

Subcutaneous tumors on the left flanks of female nude mice were initiated by injection of $5.0 \times 10^{6}$ viable cells in a volume of $0.1 \mathrm{~mL}$. Tumors were allowed to grow for approximately 7 days to become palpable. Tumor-bearing mice were randomly assigned to one of the following groups $(n=10)$ : saline, DOX, L-DOX, and Lac-L-DOX ( $5 \mathrm{mg} / \mathrm{kg})$. The mice were treated every 4 days. Animal weight and tumor volume were measured twice or three times weekly until the tumor volume reached $1500 \mathrm{~mm}^{3}$. The survival time of each mouse was then recorded, and the animals were sacrificed. Tumor volume was determined by measuring three orthogonal diameters of the tumor and was calculated as one-half of the product of the diameters. The increase in median survival was calculated as $(\mathrm{T} / \mathrm{C}-1) \times 100 \%$, where $\mathrm{T}$ is the median survival days of treated mice and $\mathrm{C}$ is the median survival days of the control mice. Animal survival was evaluated by Kaplan-Meier analysis and increased life span (ILS, \%) was calculated by ILS $=$ (mean survival time of the treated mice/mean survival time of control mice -1$) \times 100 \%$.

\section{Data analysis}

The data are presented as the mean \pm standard deviation and were analyzed by a two-tailed Student's $t$-test using MiniTAB (Minitab Inc, State College, PA). Minimal statistical significance was defined at $P<0.05$. Statistical analysis of the survival data was performed using the log-rank test.

\section{Results \\ Characterization of liposomes}

Lac-L-DOX had a mean diameter of $96 \pm 39 \mathrm{~nm}$ and a zeta potential of $-21.5 \pm 1.7 \mathrm{~nm}$, whereas L-DOX had a relatively larger diameter of $124 \pm 49 \mathrm{~nm}$ and a lower zeta potential of $-17.1 \pm 0.7 \mathrm{~nm}$. The entrapment efficiency of the liposomes by remote loading was greater than $95 \%$ at a drug to lipid weight ratio of $1: 20$.

\section{Cellular uptake of Lac-L-calcein}

The cellular uptake of Lac-L-calcein in HepG2 cells was analyzed by flow cytometry and by confocal microscopy. L-calcein was used as a nontargeted control. As shown in Figure 2, the fluorescence intensity in cells treated with Lac-L-calcein was significantly higher than that of nontargeted liposomes, and the uptake of Lac-L-calcein by HepG2 cells was decreased by treatment with $20 \mathrm{mM}$ lactose as an ASGPR-blocking agent. The results indicate that Lac-liposomes are able to target hepatocellular carcinoma cells with high expression of ASGPR.

Cellular uptake of the Lac-L-calcein and L-calcein was further evaluated by confocal microscopy (Figure 3 ). The fluorescence intensity of cells incubated with Lac-L-calcein 

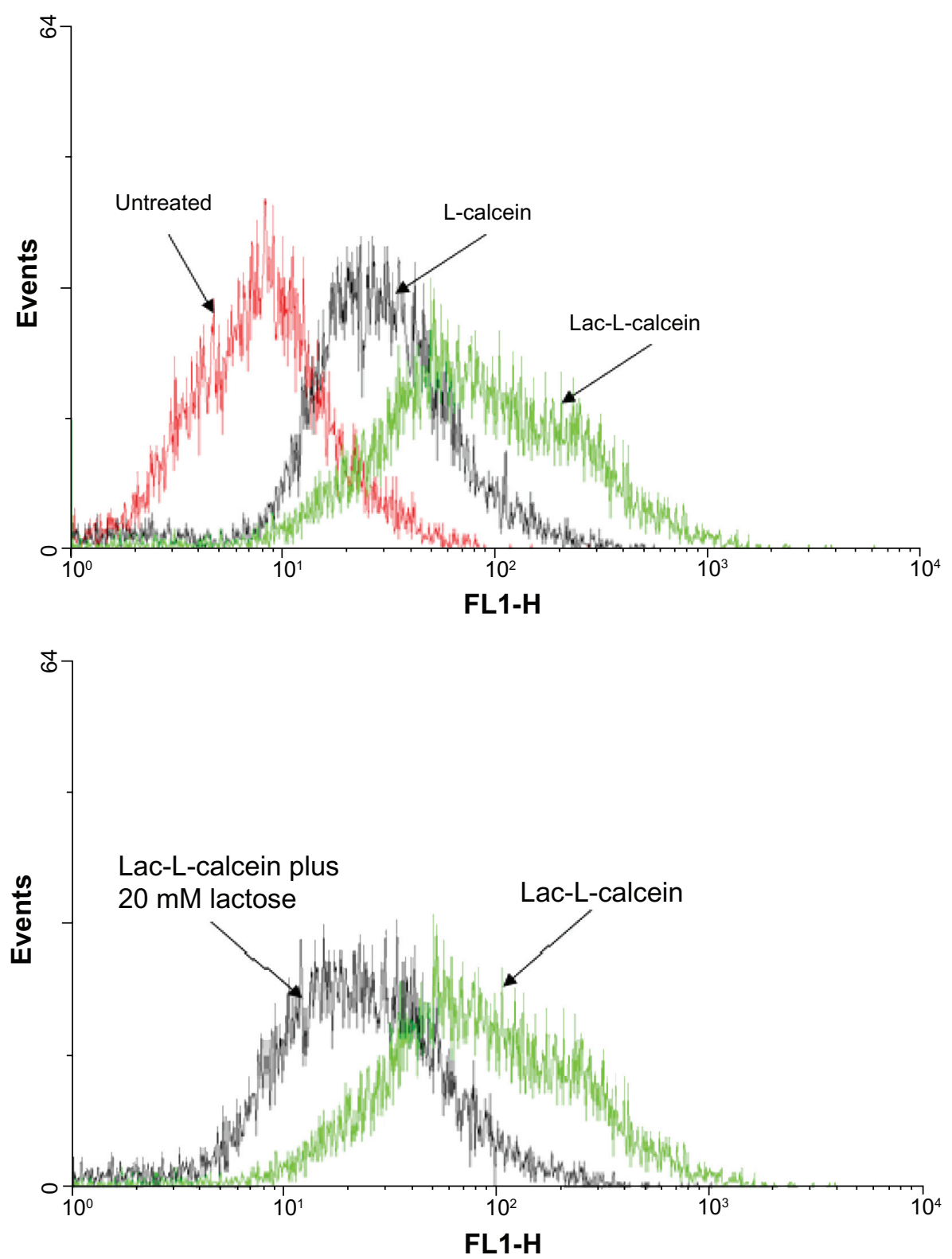

Figure 2 Cellular uptake of liposomes in HepG2 cells.

Notes: Cells were incubated with L-calcein, Lac-L-calcein, and Lac-L-calcein plus $20 \mathrm{mM}$ lactose as a blocking agent for one hour before being subjected to analysis. The fluorescence intensity in HepG2 cells treated with Lac-L-calcein was significantly higher than that of nontargeted liposomes. Meanwhile, the fluorescence of Lac-L-calcein in HepG2 cells was decreased by treatment of $20 \mathrm{mM}$ lactose as an asialoglycoprotein receptor blocking agent.

was much higher than that of cells incubated with L-calcein. Moreover, the targeting effect could be blocked by the addition of $20 \mu \mathrm{M}$ lactose, which confirmed the selectivity of lactosylated liposomal cellular uptake by ASGPR.

\section{Cytotoxicity of Lac-L-DOX}

The cytotoxicity of Lac-L-DOX in HepG2 cells was studied using MTT assay. The cells were treated with different DOX formulations. The half-maximal inhibitory concentration $\left(\mathrm{IC}_{50}\right)$ values of free DOX, L-DOX, and Lac-L-DOX in HepG2 cells are shown in Table 1. Lac-L-DOX showed the highest toxicity, and the cytotoxicity of Lac-L-DOX was significantly greater than that of L-DOX. In addition, the cytotoxicity of Lac-L-DOX was significantly reduced by blocking with $20 \mu \mathrm{M}$ lactose in HepG2 cells.

\section{Pharmacokinetic parameters of Lac-L-DOX}

The plasma concentrations of DOX after intravenous administration of Lac-L-DOX, L-DOX, and free DOX are shown in Figure 4. Pharmacokinetic parameters are summarized in Table 2. The results show that the free DOX was rapidly 

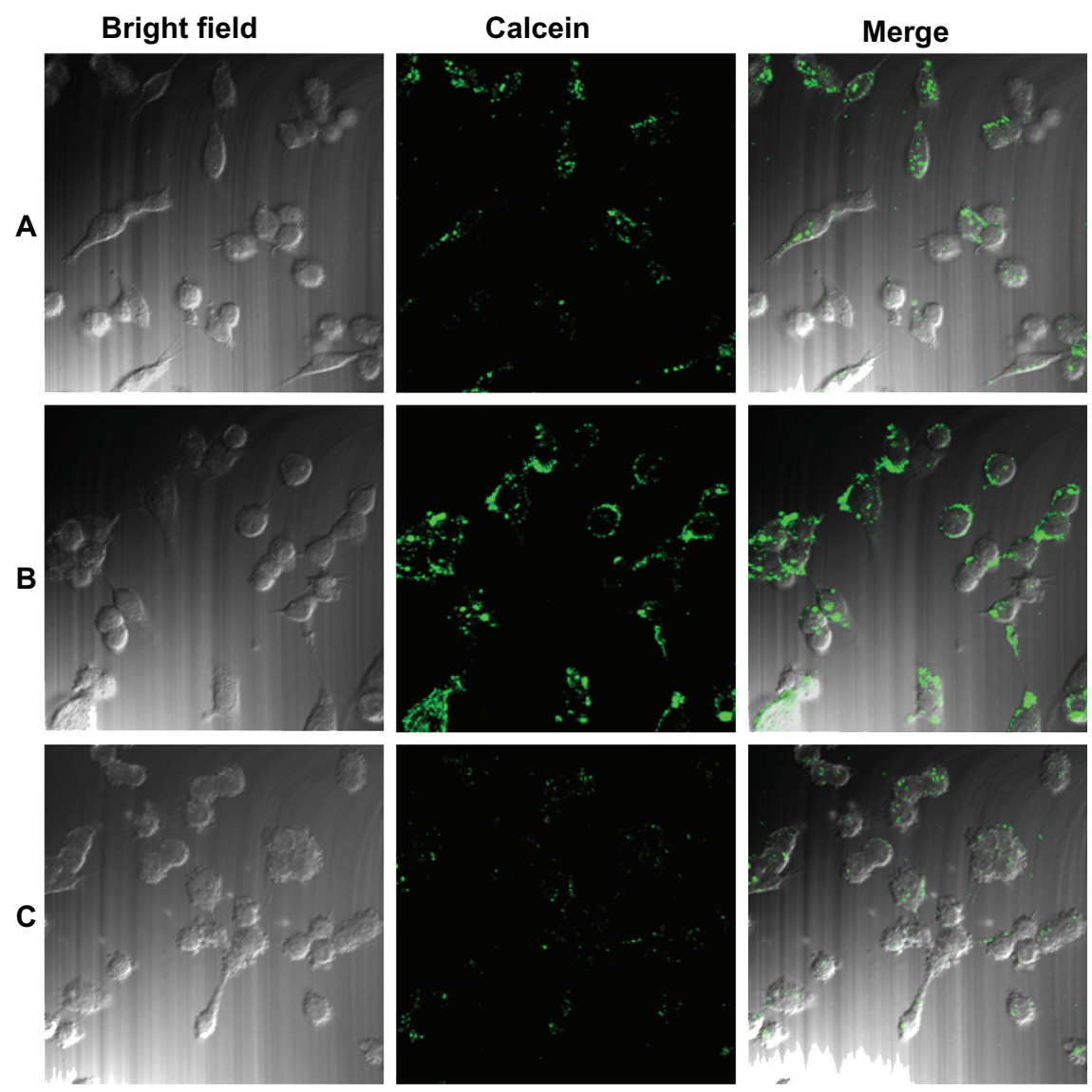

Figure 3 Cellular uptake of Lac-L-calcein in HepG2 cells detected by confocal microscopy. Cells were incubated with phosphate-buffered saline, L-calcein, Lac-L-calcein, and Lac-L-calcein plus $20 \mathrm{mM}$ lactose at the same concentrations of calcein for one hour. (A) Cells treated with L-calcein. (B) Cells treated with Lac-L-calcein. (C) Cells treated with Lac-L-calcein plus $20 \mathrm{mM}$ lactose.

eliminated, and had a relatively low half-life $\left(\mathrm{t}_{1 / 2}\right)$ and area under the concentration-time curve (AUC). Both liposomal formulations, including Lac-L-DOX and L-DOX, had a prolonged blood circulation time due to incorporation of PEG-DSPE in DOX liposomes. Meanwhile, the Lac-L-DOX was cleared more rapidly than L-DOX, suggesting that liposomes modified with Lac-DOPE have significant effects on the pharmacokinetics of L-DOX.

Table I Cytotoxicity of L-DOX formulations to HepG2 cells

\begin{tabular}{ll}
\hline & IC $_{50}(\mu \mathrm{M})$ \\
\hline Free DOX & $5.3 \pm 1.4$ \\
L-DOX & $7.5 \pm 2.8$ \\
Lac-L-DOX & $2.7 \pm 1.2^{\mathrm{a}, \mathrm{b}}$ \\
Lac-L-DOX plus $20 \mathrm{mM}$ Lactose & $9.0 \pm 2.0$ \\
\hline
\end{tabular}

Notes: Cytotoxicity was determined using the MTT assay as described in the Methods section. Data are represented as the mean \pm standard deviation $(n=3)$. ap $<0.05$ compared with L-DOX treatment; ${ }^{b} P<0.01$ compared with Lac-L-DOX plus $20 \mathrm{mM}$ lactose treatment.

Abbreviations: DOX, doxorubicin; L-DOX, liposomal doxorubicin; Lac-L-DOX, lactosylated liposomes encapsulating doxorubicin; $\mathrm{IC}_{50}$, half-maximal inhibitory concentration.

\section{Biodistribution in tumor-bearing mice}

Tissue distribution (Figure 5) showed that the free DOX accumulated mainly in the heart and its concentration in the liver and in tumor was very low. In contrast, the concentration of L-DOX in the heart was significantly lower than that of free DOX. The high DOX concentration in the heart is the primary factor contributing to its inherent cardiac toxicity. ${ }^{23}$ Moreover, the DOX concentrations of Lac-L-DOX in the liver and tumor were significantly higher than that of free DOX and L-DOX $(P<0.05$ or $P<0.005)$. Accumulation of the Lac-L-DOX in the liver was 3.4 times higher than that of free DOX and 1.5 times higher than that of L-DOX. Accumulation of Lac-L-DOX in tumor tissue was 9.8 times higher than that of free DOX and 2.4 times higher than that of L-DOX. These results indicate that incorporation of LacDOPE in liposomes results in a greater amount of DOX accumulation in liver and hepatic tumor tissue.

In order to confirm further the cellular distribution of DOX in the liver and tumor, liver sections and hepatic tumor sections were labeled with Alexa Fluor 488 conjugated with 


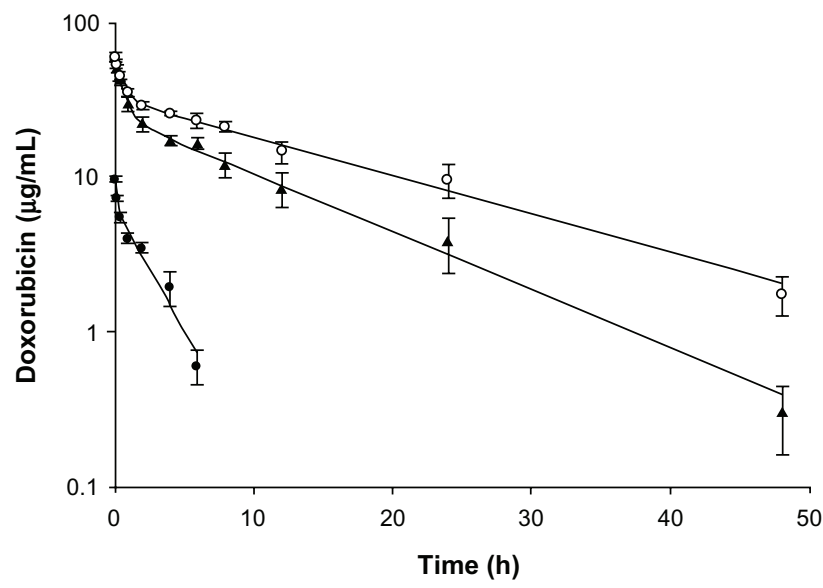

Figure 4 Plasma concentrations of DOX in different formulations.

Notes: DOX in different formulations $(5 \mathrm{mg} / \mathrm{kg}$ ) was injected via the tail veins of normal mice. Data are expressed as the mean \pm standard deviation $(n=3)$. (•) Free DOX, (०) L-DOX, ( $\mathbf{\Delta})$ Lac-L-DOX.

Abbreviations: DOX, doxorubicin; L-DOX, liposomal doxorubicin; Lac-L-DOX, lactosylated liposomes encapsulating doxorubicin.

phalloidin and observed by laser confocal scanning microscopy. As shown in Figure 6, at 4 hours after injection of free DOX, the red fluorescence density of DOX is very low and nearly invisible in tumor tissue. In contrast, the liver sections and tumor sections of L-DOX-treated mice have significantly enhanced red fluorescence density of DOX compared with that of free DOX and DOX. These results are consistent with the biodistribution of different DOX formulations as shown above and provide evidence that improved liver and tumor uptake of DOX via Lac-L-DOX is due to ASGPR.

\section{Antitumor activity of Lac-L-DOX}

The antitumor activity was evaluated in HepG2 tumor-bearing mice after intravenous injection four times every 4 days at a DOX dose of $5 \mathrm{mg} / \mathrm{kg}$ body weight of free DOX, L-DOX, and Lac-L-DOX. As depicted in Figure 7, compared with the PBS group, all of the DOX formulations inhibited tumoral growth.

Table 2 Pharmacokinetic parameters in different formulations after intravenous injection at a doxorubicin dose of $5 \mathrm{mg} / \mathrm{kg}$ body weight $(n=3)$

\begin{tabular}{lccc}
\hline & Free DOX & \multicolumn{1}{l}{ L-DOX } & Lac-L-DOX \\
\hline AUC $(\mu$ g hours $/ \mathrm{mL})$ & $16.83 \pm 2.7$ & $582.3 \pm 27.3$ & $350.2 \pm 20.2^{\mathrm{a}, \mathrm{b}}$ \\
$\mathrm{t}_{1 / 2}$ (hours) & $1.96 \pm 0.7$ & $12.08 \pm 0.8$ & $8.73 \pm 0.8^{\mathrm{a}, \mathrm{b}}$ \\
$\mathrm{CL}$ (mL/hour) & $11.33 \pm 1.0$ & $0.086 \pm 0.0$ & $0.014 \pm 0.0^{\mathrm{a}, \mathrm{b}}$ \\
$\mathrm{MRT}$ (hours) & $2.56 \pm 0.8$ & $16.9 \pm 1.1$ & $11.8 \pm 1.1^{\mathrm{a}, \mathrm{b}}$ \\
\hline
\end{tabular}

Notes: ${ }^{p} P<0.01$ compared with free doxorubicin; ${ }^{b} P<0.01$ compared with L-DOX. Abbreviations: $A \cup C$, the area under the concentration-time curve; $t_{1 / 2}$, half life; $\mathrm{CL}$, total body clearance in mice; MRT, mean residence time; DOX, doxorubicin; L-DOX, liposomal doxorubicin; Lac-L-DOX, lactosylated liposomes encapsulating doxorubicin.

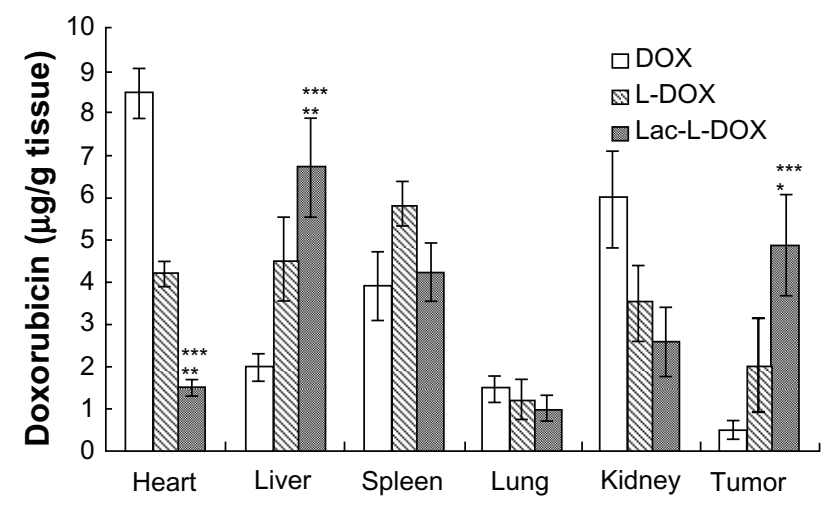

Figure 5 Tissue distribution of DOX in different formulations at 4 hours after intravenous injection in female tumor-bearing nude mice.

Notes: Data are expressed as the mean \pm standard deviation $(n=5)$. *** $p<0.005$, significant difference compared with free DOX; $* * P<0.005$, significant difference compared with L-DOX; $* P<0.05$, significant difference compared with L-DOX

Abbreviations: DOX, doxorubicin; L-DOX, liposomal doxorubicin; Lac-L-DOX, lactosylated liposomes encapsulating doxorubicin.

Notably, treatment with Lac-L-DOX resulted in significantly greater growth retardation of HepG2 tumors compared with L-DOX and free DOX.

The antitumor effect of Lac-L-DOX were also demonstrated by survival time in nude mice bearing HepG2 xenografts. As shown in Figure 8 and Table 3, median survival times in the Lac-L-DOX, L-DOX, free DOX, and PBS groups were 70, 42, 34 , and 28 days, respectively. The corresponding percentage increase in life span among mice treated with Lac-L-DOX, L-DOX, and free DOX based on median survival times was $150 \%, 50 \%$, and $21.4 \%$, respectively. Compared with mice treated with PBS and those treated with L-DOX, mice treated with Lac-L-DOX $(P<0.001$ or $P<0.05$, log-rank analysis $)$ had a significantly prolonged survival time. The high antitumor activity of Lac-L-DOX suggests that it accumulates in tumor tissue due to recognition of the galactosyl residues of LacDOPE by ASGPR on the surface of the hepatic tumor. ${ }^{26}$

\section{Discussion}

Liposomes have been widely studied as drug carriers for increasing the therapeutic index of chemotherapeutics. Liposomes are cleared from the blood circulation by the RES within a few minutes to a few hours. ${ }^{27}$ In this study, PEGylated liposomes were chosen because liposomes modified by PEG-lipid increase the blood circulation time and reduce uptake by the RES. ${ }^{28,29}$

Some articles have reported that prolonged circulation of liposomes can enhance drug therapeutic efficacy via the enhanced permeation and retention effect within tumor tissue. ${ }^{30,31}$ However, Hong et al ${ }^{22}$ previously reported that in liposome systems, surface coating with PEG offered no 

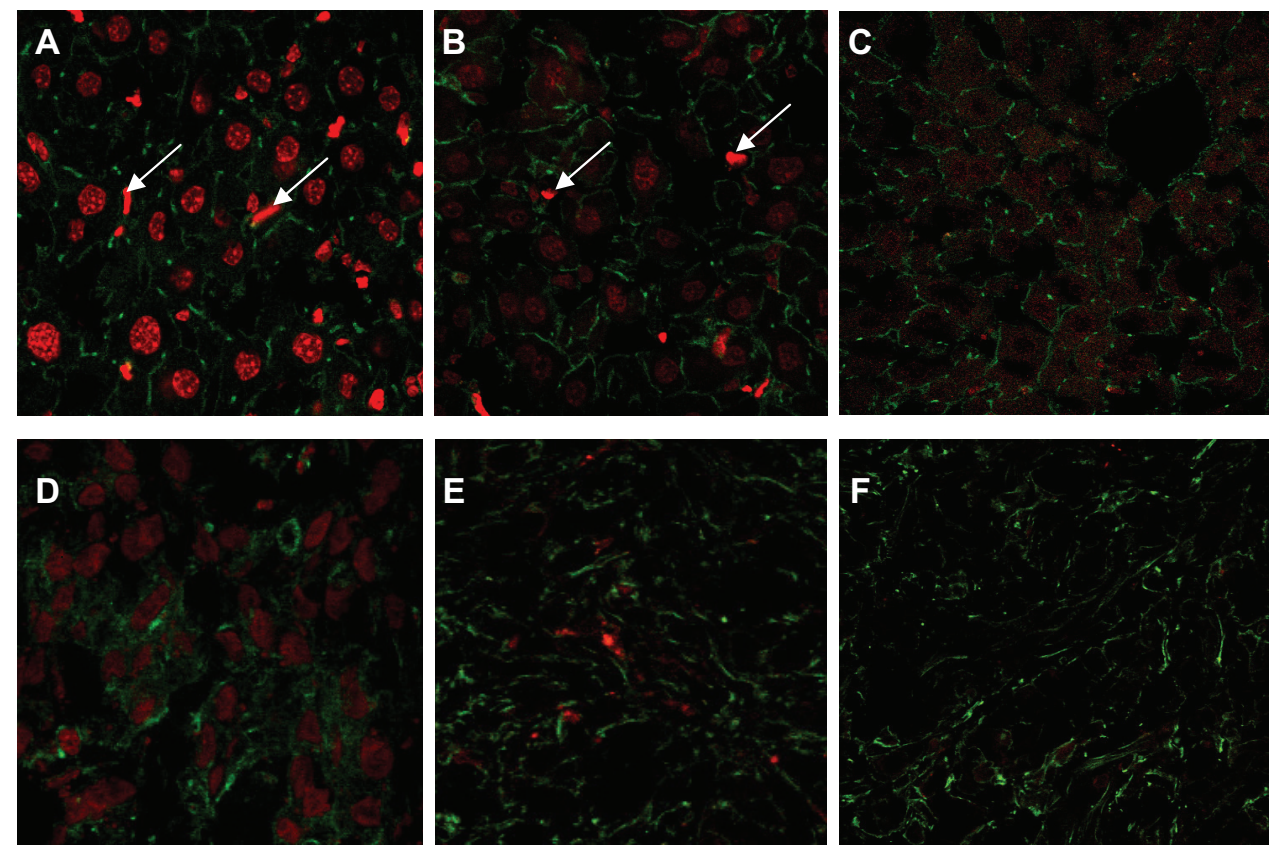

Figure 6 Confocal micrographs of tissue from female tumor-bearing nude mice injected with different formulations of DOX, 4 hours prior to euthanasia. The tissue section was labeled for cytoskeletal filamentous actin with Alexa Fluor $488^{\circledR}$ conjugated with phalloidin. (A) Lac-L-DOX in liver, (B) L-DOX in liver, (C) free DOX in liver, (D) LacL-DOX in tumor, (E) L-DOX in tumor, and (F) free DOX in tumor.

Note: The white arrows denote Kupffer cells.

Abbreviations: DOX, doxorubicin; L-DOX, liposomal doxorubicin; Lac-L-DOX, lactosylated liposomes encapsulating doxorubicin.

benefit for L-DOX in the C-26 tumor model. Therefore, simply increasing the plasma AUC by surface coating with PEG may not be sufficient to enhance the therapeutic index of drugs. For a variety of chemotherapeutic agents with an intracellular site of action, efficient intracellular uptake by tumor cells is the critical step in determining antitumor activity.

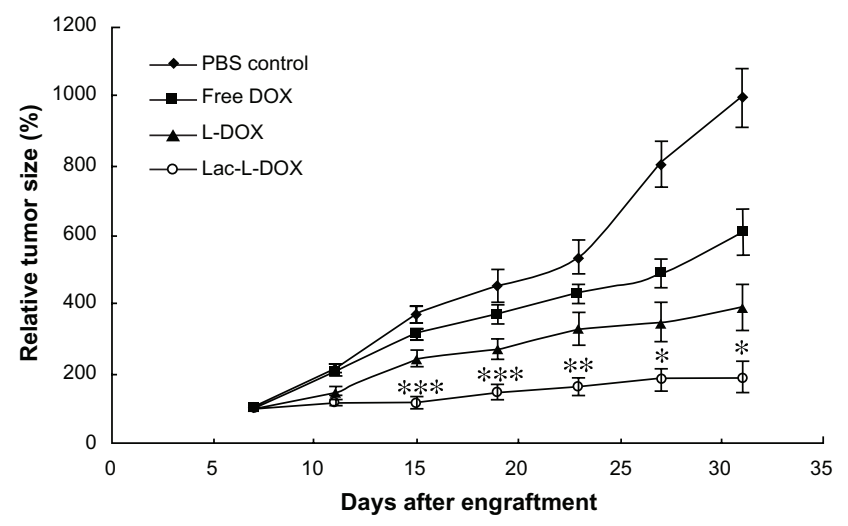

Figure 7 Tumor growth inhibition after intravenous injection of free DOX, L-DOX, or Lac-L-DOX in tumor-bearing mice at a DOX dose of $5 \mathrm{mg} / \mathrm{kg}$ body weight. Notes: Data are shown as the mean \pm standard deviation $(n=10)$. **** $<0.05$, statistically significant compared with phosphate-buffered saline control; **P $<0.05$, statistically significant compared with free DOX; $* P<0.05$, statistically significant compared with L-DOX.

Abbreviations: DOX, doxorubicin; L-DOX, liposomal doxorubicin; Lac-L-DOX, lactosylated liposomes encapsulating doxorubicin; PBS, phosphate-buffered solution.
Ligand-mediated targeting for drug delivery systems is one of the most exciting areas because it can improve site-specific drug delivery. Many attempts have been made to deliver drugs specifically to liver cells for effective treatment of liver diseases. Among the various receptors on parenchymal and nonparenchymal cells, the ASGPR is the most promising receptor site because of its high affinity and rapid internalization. ${ }^{32}$ Targeting ASGPR is an attractive strategy for liver-specific delivery due to its exclusive expression by parenchymal hepatocytes. ${ }^{33}$ In order to achieve highly effective hepatic tumor-targeting delivery systems for chemotherapeutic agents, a lactosylated lipid, Lac-DOPE, was synthesized and a liposome modified with Lac-DOPE and MPEG-DSPE was designed and evaluated for targeting and delivery efficiency.

In the uptake and cytotoxicity studies, lactose was chosen as the ASGPR-blocking agent because it can specifically recognize and bind ASGPR. $.34,35$ The cytotoxicity of different DOX formulations was studied by MTT assay, which measures mitochondrial function or integrity.

DOX proved to be an excellent compound for demonstration of targeted drug delivery. The fluorescence properties of DOX allow for quantitative measurement of biodistribution both by determining the DOX concentration in tissues using fluorescence spectroscopy, as well as by fluorescence 


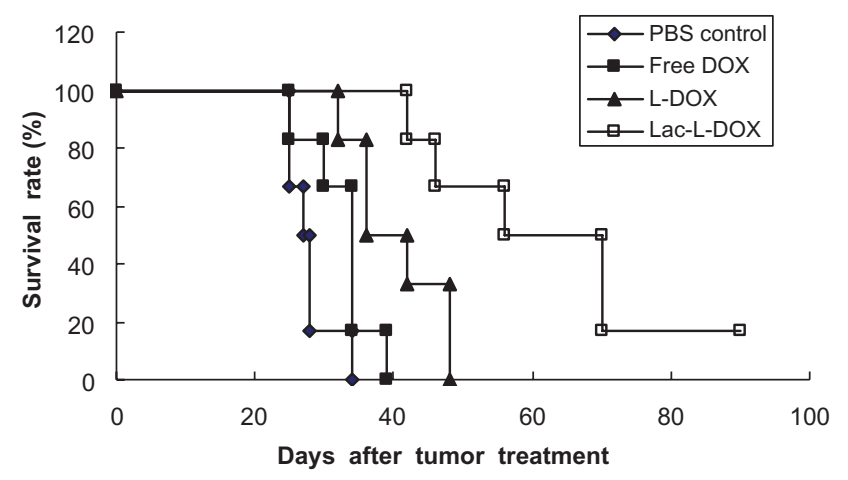

Figure 8 Kaplan-Meier survival curve of mice bearing HepG2 xenografts. Notes: Animals (10 per group) were treated with $5 \mathrm{mg} / \mathrm{kg}$ of free DOX, L-DOX or Lac-L-DOX. The treatment was continued every 4 days for four times.

Abbreviations: DOX, doxorubicin; L-DOX, liposomal doxorubicin; Lac-L-DOX, lactosylated liposomes encapsulating doxorubicin; PBS, phosphate-buffered solution.

microscopy of fixed tissue sections. ${ }^{36}$ In order to show hepatic tumor targeting of Lac-L-DOX, the biodistribution of DOX in different formulations was determined and the red fluorescence images of DOX in liver and tumor were studied by confocal microscopy. The biodistribution study results suggest that Lac-L-DOX allows higher DOX concentrations to be reached in both normal liver and hepatic tumor tissue than do L-DOX and free DOX. The fluorescence images of liver sections showed much more DOX associated with hepatocyte nuclei than Kupffer cell nuclei. DOX exerts its antitumor activity after intercalating with double-stranded DNA in nuclei. ${ }^{37}$ Thus, intracellular delivery of DOX into tumor cells will be essential to its antitumor activity. On the other hand, in the heart, Lac-L-DOX showed a lower drug concentration than L-DOX and free DOX. This result suggests that the lactosylated liposome has selective hepatic tumor targeting.

Consistent with its higher accumulation in tumor tissue, Lac-L-DOX had stronger tumor growth inhibition activity

Table 3 Survival of mice after treatment with free DOX, L-DOX, and Lac-L-DOX in HepG2 tumor-bearing mice ${ }^{a}$

\begin{tabular}{lllll}
\hline Treatment & $\begin{array}{l}\text { Tumor } \\
\text { inhibition } \\
\text { ratio }^{\mathbf{b}}(\%)\end{array}$ & $\begin{array}{l}\text { MST }^{\mathbf{c}} \\
(\text { days) }\end{array}$ & $\begin{array}{l}\text { ILS }^{\mathbf{d}} \\
(\%)\end{array}$ & $\begin{array}{l}\text { Log-rank } \\
\text { P value }\end{array}$ \\
\hline PBS & 0 & 28 & 0 & - \\
Free DOX & 38.7 & 34 & 21.4 & 0.0612 \\
L-DOX & 60.8 & 42 & 50 & 0.00178 \\
Lac-L-DOX & 80.9 & 70 & 150 & 0.000557 \\
& & & & $(0.0205)^{\mathrm{e}}$ \\
\hline
\end{tabular}

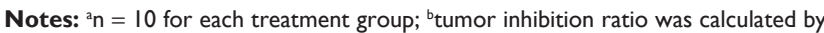
$(\mathrm{C}-\mathrm{T}) / \mathrm{C} \times 100 \%$; $\mathrm{CMST}$ represents the mean survival time; 'ILS (\%) was calculated by (MST of the treated mice/MST of control mice -1 ) $\times 100 \%$; $P$ value was calculated in comparison with L-DOX.

Abbreviations: MRT, mean residence time; DOX, doxorubicin; L-DOX, liposomal doxorubicin; Lac-L-DOX, lactosylated liposomes encapsulating doxorubicin; MST, mean survival time; ILS, increased life span; PBS, phosphate-buffered saline. than L-DOX and free DOX, which may be explained by the following: lactosylation of the liposomes enhanced cellular uptake and internalization of DOX into hepatoma cells via ASGPR-mediated pathways; PEGylation of liposomes increased accumulation of DOX in tumor tissue by prolonging the circulation time and reducing uptake by the RES; the small particle size of Lac-L-DOX enables easier penetration of the tumor via the enhanced permeation and retention effect. In summary, a novel lactosylated liposomal vehicle modified with Lac-DOPE was developed. This novel lactosylated formulation is promising as a targeted carrier for chemotherapeutic agents against hepatic tumor and warrants further investigation.

\section{Acknowledgments}

This work was supported in part by the China Scholarship Council (20103023).

\section{Disclosure}

The authors report no conflicts of interest in this work.

\section{References}

1. El-Serag HB, Rudolph L. Hepatocellular carcinoma: epidemiology and molecular carcinogenesis. Gastroenterology. 2007;132:2557-2576.

2. Gabizon A, Dagan A, Goren D, Barenholz Y, Fuks Z. Liposomes as in vivo carriers of adriamycin: reduced cardiac uptake and preserved antitumor activity in mice. Cancer Res. 1982;42:4734-4739.

3. Safra T, Muggia F, Jeffers $S$, et al. Pegylated liposomal doxorubicin (doxil): reduced clinical cardiotoxicity in patients reaching or exceeding cumulative doses of $500 \mathrm{mg} / \mathrm{m}^{2}$. Ann Oncol. 2000;11:1029-1033.

4. Yan X, Scherphof GL, Kamps JA. Liposome opsonization. J Liposome Res. 2005;15:109-139.

5. Hashida M, Nishikawa M, Takakura Y. Hepatic targeting of drugs and proteins by chemical modification. $J$ Control Release. 1995;36:99-107.

6. Rensen PC, Sliedregt LA, Ferns M, et al. Determination of the upper size limit for uptake and processing of ligands by the asialoglycoprotein receptor on hepatocytes in vitro and in vivo. $J$ Biol Chem. 2001;276:37577-37584.

7. Seymour LW, Ferry DR, Anderson D, et al. Hepatic drug targeting: phase 1 evaluation of polymer-bound doxorubicin. $J$ Clin Oncol. 2002;20:1668-1676.

8. Wu J, Nantz MH, Zern MA. Targeting hepatocytes for drug and gene delivery: emerging novel approaches and applications. Front Biosci. 2002;7:717-725.

9. Suo A, Qian J, Yao Y, Zhang W. Galactosylated poly(ethylene glycol)b-poly (L-lactide-co- $\beta$-malic acid) block copolymer micelles for targeted drug delivery: preparation and in vitro characterization. Int $J$ Nanomedicine. 2010;5:1029-1038.

10. Managit C, Kawakami S, Yamashita F, Hashida M. Effect of galaclactose density on asialoglycoprotein receptor-mediated uptake of galactosylated liposomes. J Pharm Sci. 2005;94:2266-2275.

11. Hattori Y, Kawakami S, Yamashita F, Hashida M. Controlled biodistribution of galactosylated liposomes and incorporated probucol in hepatocyte-selective drug targeting. $J$ Control Release. 2000;69: 369-377.

12. Managit C, Kawakami S, Yamashita F, Hashida M. Uptake characteristics of galactosylated emulsion by HepG2 hepatoma cells. Int J Pharm. 2005;301:255-261. 
13. Wang $\mathrm{S}, \mathrm{Xu} \mathrm{H}, \mathrm{Xu}$ J, et al. Synthesis of a novel galactosylated lipid and its application to the hepatocyte-selective targeting of liposomal doxorubicin. Eur J Pharm Biopharm. 2006;62:32-38.

14. Terada T, Iwai M, Kawakami S, Yamashita F, Hashida M. Novel PEGmatrix metalloproteinase-2 cleavable peptide-lipid containing galactosylated liposomes for hepatocellular carcinoma-selective targeting. J Control Release. 2006;111:333-342.

15. Xu Z, Chen L, Gu W, et al. The performance of docetaxel-loaded solid lipid nanoparticles targeted to hepatocellular carcinoma. Biomaterials. 2009;30:226-232.

16. Szoka F, Olson F, Heath T, Vail W, Mayhew E, Papahadjopoulos D. Reparation of unilamellar liposomes of intermediate size $(0.1-0.2 \mu \mathrm{M})$ by a combination of reverse phase evaporation and extrusion through polycarbonate membranes. Biochim Biophys Acta. 1980;601: 559-571.

17. Pan XQ, Wang H, Lee RJ. Antitumor activity of folate receptor-targeted liposomal doxorubicin in a $\mathrm{KB}$ oral carcinoma murine xenograft model. Pharm Res. 2003;20:417-422.

18. Lee RJ, Low PS. Folate-mediated tumor cell targeting of liposomeentrapped doxorubicin in vitro. Biochim Biophys Acta. 1995;1233: 134-144.

19. Li H, LuY, Piao L, et al. Targeting human clonogenic acute myelogenous leukemia cells via folate conjugated liposomes combined with receptor modulation by all-trans retinoic acid. Int J Pharm. 2010;402:57-63.

20. Marks DC, Belov L, Davey MW, Davey RA, Kidman AD. The MTT cell viability assay for cytotoxicity testing in multidrug-resistant human leukemic cells. Leuk Res. 1992;16:1165-1173.

21. Xu S, Liu Y, Tai HC, Zhu J, Ding H, Lee RJ. Synthesis of transferrin (Tf) conjugated liposomes via Staudinger ligation. Int J Pharm. 2011;404:205-210.

22. Hong RL, Huang CJ, Tseng YL, et al. Direct comparison of liposomal doxorubicin with or without polyethylene glycol coating in C-26 tumorbearing mice: is surface coating with polyethylene glycol beneficial? Clin Cancer Res. 1999;5:3645-3652.

23. Tagami T, Ernsting MJ, Li SD. Efficient tumor regression by a single and low dose treatment with a novel and enhanced formulation of thermosensitive liposomal doxorubicin. J Control Release. 2011;152: 303-309.

24. Laginha KM, Verwoert S, Charrois GJ, Allen TM. Determination of doxorubicin levels in whole tumor and tumor nuclei in murine breast cancer tumors. Clin.Cancer Res. 2005;11:6944-6949.
25. Han HD, Lee A, Hwang T, et al. Enhanced circulation time and antitumor activity of doxorubicin by comblike polymer-incorporated liposomes. J Control Release. 2007;120:161-168.

26. Wang S, Deng Y, Xu H, Wu H, Qiu Y, Chen D. Synthesis of a novel galactosylated lipid and its application to the hepatocyte-selective targeting of liposomal doxorubicin. Eur J Pharm Biopharm. 2006;62:32-38.

27. Allen TM. Long-circulating (sterically stabilized) liposomes for targeted drug delivery. Trends Pharmacol Sci. 1994;15:215-220.

28. Gabizon A, Papahadjopoulos D. Liposome formulations with prolonged circulation time in blood and enhanced uptake by tumors. Proc Natl Acad Sci U S A. 1988;85:6949-6953.

29. Woodle MC, Lasic DD. Sterically stabilized liposomes. Biochim Biophys Acta. 1992;1113:171-199.

30. Mayer LD, Tai LC, Ko DS, et al. Influence of vesicle size, lipid composition, and drug-to-lipid ratio on the biological activity of liposomal doxorubicin in mice. Cancer Res. 1989;49:5922-5930.

31. Gabizon AA. Selective tumor localization and improved therapeutic index of anthracyclines encapsulated in long-circulating liposomes. Cancer Res. 1992;52:891-896.

32. Jain NK, Jain SK. Development and in vitro characterization of galactosylated low molecular weight chitosan nanoparticles bearing doxorubicin. AAPS PharmSciTech. 2010;11:686-697.

33. Bernardes GJ, Kikkeri R, Maglinao M, et al. Design, synthesis and biological evaluation of carbohydrate-functionalized cyclodextrins and the liposomes for hepatocyte-specific targeting. Org Biomol Chem. 2010;8:4987-4996.

34. Kikkeri R, Lepenies B, Adibekian A, Lauorino P, Seeberger PH. In vitro imaging and in vivo liver targeting with carbohydrate capped quantum dots. J Am Chem Soc. 2009;131:2110-2112.

35. Higuchi Y, Oka M, Kawakami S, Hashida M. Mannosylated semiconductor quantum dots for the labeling of macrophages. J Control Release. 2008; 125:131-136.

36. Longmuir KJ, Haynes SM, Baratta JL, Kasabwalla N, Robertson RT. Liposomal delivery of doxorubicin to hepatocytes in vivo by targeting heparin sulfate. Int J Pharm. 2009;382:222-233.

37. Gewirtz DA. A critical evaluation of the mechanisms of action proposed for the antitumor effects of the anthracycline antibiotics adriamycin and daunorubicin. Biochem Pharmacol. 1999;57:727-741.
International Journal of Nanomedicine

\section{Publish your work in this journal}

The International Journal of Nanomedicine is an international, peerreviewed journal focusing on the application of nanotechnology in diagnostics, therapeutics, and drug delivery systems throughout the biomedical field. This journal is indexed on PubMed Central, MedLine, CAS, SciSearch $\AA$, Current Contents ${ }^{\circledR} /$ Clinical Medicine,

\section{Dovepress}

Journal Citation Reports/Science Edition, EMBase, Scopus and the Elsevier Bibliographic databases. The manuscript management system is completely online and includes a very quick and fair peer-review system, which is all easy to use. Visit http://www.dovepress.com/ testimonials.php to read real quotes from published authors. 